\title{
Analysis of the execution time of multi-stage control operations with multiple measurements
}

\author{
Vadim Kopp ${ }^{1}$, Alexey Balakin ${ }^{1, *}$, Natalya Balakina ${ }^{1}$, and Mikhail Zamoryonov ${ }^{1}$ \\ 1"Sevastopol State University", Sevastopol, Russian Federation
}

\begin{abstract}
Questions related to the management of the process of multiple measurements are considered, depending on the result, which affects their number. In this case, the measurement time can be significantly increased. The above algorithm for controlling the measurement process justifies the random nature of the duration of its execution, which entails the need to study the time of carrying out multiple measurements. The paper constructs a semi-Markov model that allows to determine the distribution functions of time between events in the output flow of products after measurements, which makes it possible to dock this model with models of elements of higher levels of the hierarchy of the production structure. In the model, it is taken into account that some part of the production after a given number of measurements is defective and leaves the system. The constructed semi-Markov model allows to predict the performance of a technical system associated with multiple measurements.
\end{abstract}

The problem of controlling the process of multiple measurements is as follows. The first measurement gives an initial estimate of the value of the monitored parameter and, depending on the proximity to the tolerance boundary, the number of multiple measurements is determined [1-8]. In subsequent measurements, the evaluation of the value of the monitored parameter is refined and, accordingly, the number of multiple measurements is refined. In view of the random nature of the error of the measuring device, cases are possible in which, for example, each subsequent measurement gives a worse result than the previous one in the sense of proximity to the tolerance boundary, which entails, respectively, an increase in the number of multiple measurements. Therefore, the measurement time can be significantly increased. In this case, it is advisable to interrupt the measurement of this product, consider it defective in the measured parameter and start the process of measuring the next product. Thus, it is advisable to specify a control number or a control time for the measurement, after which the process begins again.

The described algorithm for controlling the measurement process justifies the random nature of the duration of its execution, which entails the need to study the time of carrying out multiple measurements.

Let's consider the case when the number of repeated measurements at each $\mathrm{k}$-stage is limited and can not exceed numbers $\mathrm{m}$, and the times of repeated measurements are

\footnotetext{
* Corresponding author: AIBalakin@sevsu.ru
} 
different and are described by the FP $F_{k}^{g}(t)$, where $k=(\overline{1, n})$ is the number of stages, and $g=(\overline{1, m})$ is the number of the repeated measurement/

In order to determine the FR of the time spent for $\mathrm{m}$ - repeated measurements, it is necessary to construct a semi-Markov process in a discrete phase space using the state graph presented in Figure 1.

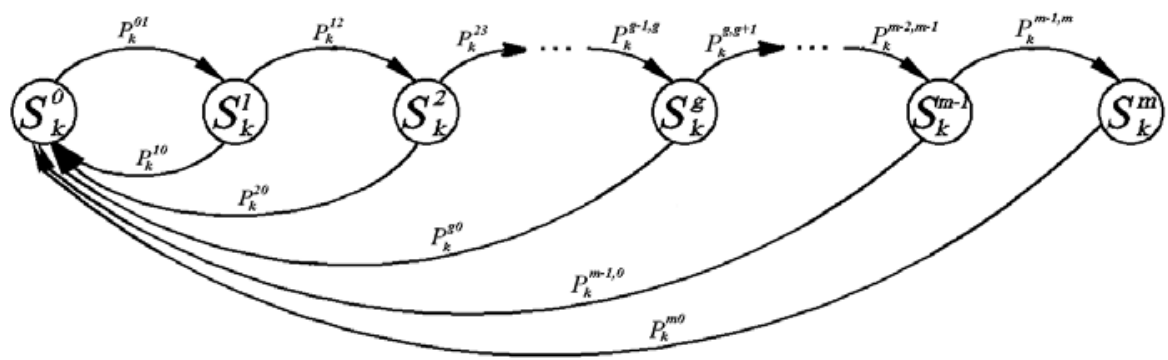

Fig. 1. The state graph of the k-th stage with $\mathrm{m}$ - limited number of repeated measurements on it

We assume that the process of Markov recovery is given by a two-component Markov chain $\left(S_{i}, \theta_{i} ; i \geq 0\right)$ with transition probabilities [9-14]:

$$
P\left\{S_{i+1}=r, \theta_{i+1} \leq t / S_{i}=h\right\}=Q_{h r}(t)
$$

In this case the components $S_{i}$ and $\theta_{i}$ are conditionally independent, that is, the equality has done

$$
P\left\{S_{i+1}=r, \theta_{i+1} \leq t / S_{i}=h\right\}=P\left\{S_{i+1}=r / S_{i}=h\right\} \cdot P\left\{\theta_{i+1} \leq t / S_{i}=h\right\} .
$$

Then the components of the semi-Markov matrix for the discrete phase space of states $E=\overline{(1, i)}$ are:

$$
Q_{h r}(t)=P_{h r} G_{h}(t), \quad h, r \in E,
$$

where $\sum_{r \in E} Q_{h r}(t)=P\left\{\theta_{i+1} \leq t / S_{i}=h\right\}=G_{h}(t), h \in E$.

We define the stationary distribution $\pi=\left(\pi_{k}^{g} ; g \in E\right)$ of a semi-Markov process (SMP) in phase space $E$ by the formulas given in [9]:

$$
\begin{array}{r}
\pi_{k}^{g}=\rho_{k}^{q} M_{k}^{q} / M ; \\
M=\sum_{g \in E} \rho_{k}^{g} M_{k}^{g}, g \in E,
\end{array}
$$

where $\rho=\left(\rho_{k}^{g} ; g \in E\right)$ - stationary distribution of nested Markov chains (NMC) $\left(S_{n} ; n \geq 0\right) ; M_{k}^{g}=M\left(v_{k}^{g}\right)$ - mean residence times in NMC; $M_{k}^{g}$ - mathematical expectation of time of stay in states $g \in E$.

Values $\rho_{k}^{g}$ are determined from the system of equations [9]: 


$$
\rho_{k}^{g}=\sum_{r \in E} \rho_{k}^{r} P_{k}^{r g}
$$

where $\sum_{g} \rho_{k}^{g}=1$.

Graph states:

$S_{k}^{0}$ - instantaneous state, corresponding to the moment of the end of the measurement process in the previous stage (note that probability $P_{k}^{01}=1$ );

$S_{k}^{1}$ - the first dimension with the distribution function $F_{k}^{1}(t)$ and mathematical expectation $M_{k}^{1}$;

$S_{k}^{2}$ - the second dimension with the distribution function $F_{k}^{2}(t)$ and mathematical expectation $M_{k}^{2}$;

$S_{k}^{g}$ - the g-th dimension with the distribution function and the mathematical expectation; ... - $S_{k}^{m}$ the m-th dimension with the distribution function $F_{k}^{g}(t)$ and the mathematical expectation $M_{k}^{g}$.

Let us, on the basis of (2), construct a system of equations for determining $\rho_{k}^{g}, g=(\overline{0, m})$ :

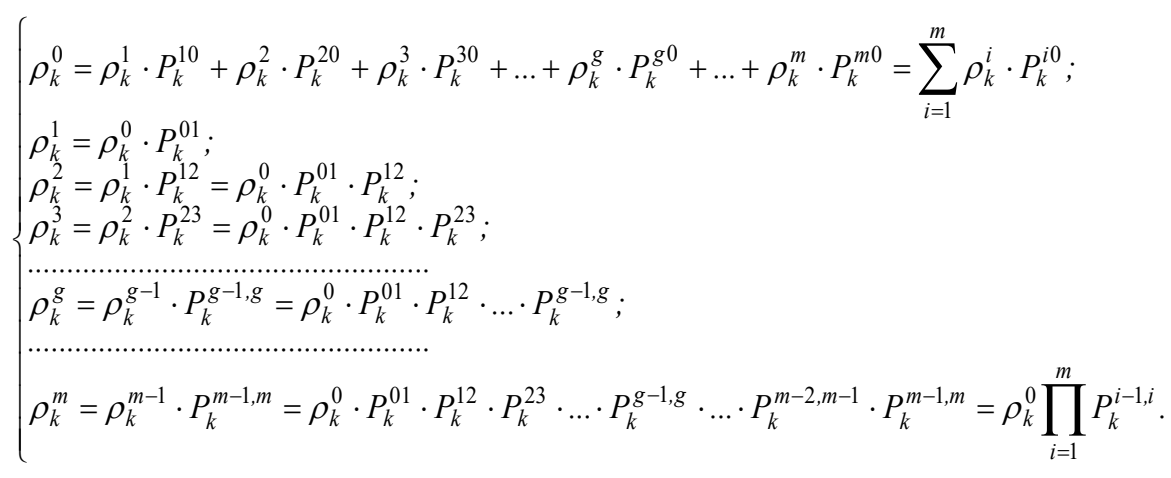

The normalization condition

$$
\sum_{g=1}^{m} \rho_{k}^{g}=1 .
$$

From the graph (Figure 1) we see that,

$$
\left\{\begin{array}{l}
P_{k}^{10}=P_{k}^{20}=P_{k}^{30}=\ldots=P_{k}^{g 0}=\ldots=P_{k}^{m-1,0}=p_{k} ; \\
P_{k}^{m 0}=1 ; \\
P_{k}^{01}=1 ; \\
P_{k}^{12}=P_{k}^{23}=\ldots=P_{k}^{g-1, g}=\ldots=P_{k}^{m-1, m}=\left(1-p_{k}\right) .
\end{array}\right.
$$

Substituting all the values for $\rho_{k}^{g}, g=(\overline{0, m})$ from (3) into the normalization condition (4), we obtain 


$$
\rho_{k}^{0}\left(1+P_{k}^{01}+P_{k}^{01} \cdot P_{k}^{12}+P_{k}^{01} \cdot P_{k}^{12} \cdot P_{k}^{23}+\ldots P_{k}^{01} \cdot P_{k}^{12} \cdot P_{k}^{23} \cdot \ldots \cdot P_{k}^{g-1, g}+\ldots+\prod_{i=1}^{m} P_{k}^{i-1,1}\right)=1
$$

where

$$
\rho_{k}^{0}=\left(1+P_{k}^{01}+P_{k}^{01} \cdot P_{k}^{12}+P_{k}^{01} \cdot P_{k}^{12} \cdot P_{k}^{23}+\ldots+\prod_{i=1}^{m} P_{k}^{i-1, i}\right)^{-1}
$$

Substituting expressions (5) in (3), (6) we finally obtain:

$$
\left\{\begin{array}{l}
\rho_{k}^{0}=\left[2+\left(1-p_{k}\right)+\left(1-p_{k}\right)^{2}+\ldots+\left(1-p_{k}\right)^{m-1}\right]^{-1} \\
\rho_{k}^{1}=\rho_{k}^{0} \\
\rho_{k}^{2}=\rho_{k}^{0} \cdot\left(1-p_{k}\right) \\
\rho_{k}^{3}=\rho_{k}^{0} \cdot\left(1-p_{k}\right)^{2} \\
\cdots \cdots \cdots \cdots \cdots \cdots \cdots \cdots \\
\rho_{k}^{g}=\rho_{k}^{0} \cdot\left(1-p_{k}\right)^{g-1} \\
\cdots \ldots \ldots \ldots \ldots \ldots \ldots \ldots . . \\
\rho_{k}^{m}=\rho_{k}^{0} \cdot\left(1-p_{k}\right)^{m-1}
\end{array}\right.
$$

On the basis of (1), (7) we determine the stationary distribution of SMP

$$
\begin{gathered}
M=\sum_{i=0}^{m} \rho_{k}^{i} \cdot M_{k}^{i}=\sum_{i=0}^{m}\left[\rho_{k}^{0}(1-p)^{i-1}\right] \cdot M_{k}^{i}+\rho_{k}^{0} \cdot M_{k}^{0} \\
\pi_{k}^{i}=\frac{\rho_{k}^{i} \cdot M_{k}^{i}}{M} .
\end{gathered}
$$

We will solve the problem of determining the time phase of the measurement at the stage using the formula of total probability.

To do this, we accept the following hypotheses: $H_{1}$ - the first measurement is performed; ... $H_{n}$ - The $\mathrm{n}$-th dimension is being implemented.

The probabilities of these hypotheses, with regard for (8), are equal to

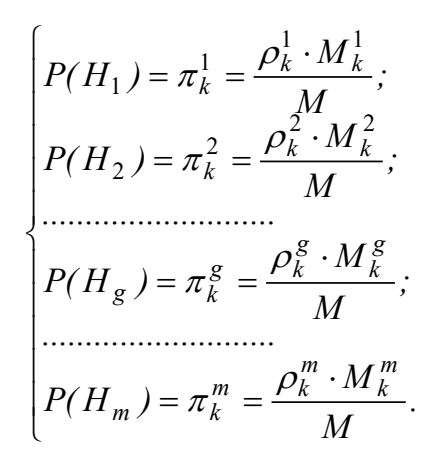

We introduce an $A$ - event, by which we will understand the duration of the measurement at the stage. The conditional probability of an $A$ - event when the hypotheses $H_{1}, \ldots, H_{n}$ are fulfilled is equal to 


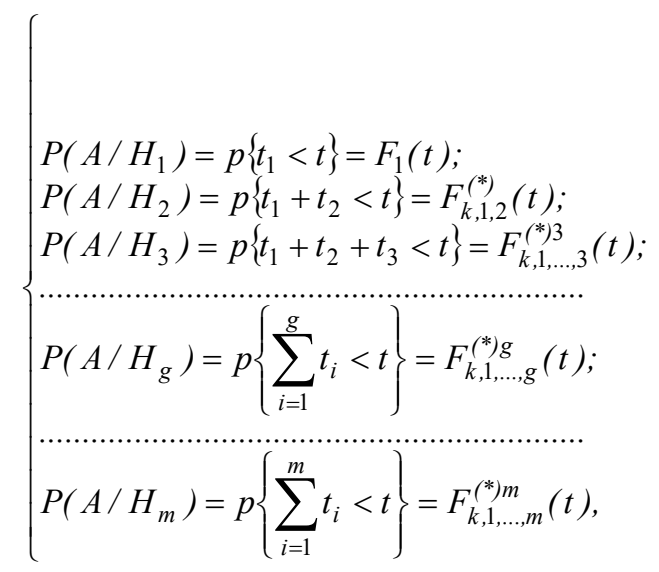

where $\left(^{*}\right) m$ is the notation for a multiple $\mathrm{m}$-fold operation.

The function of the distribution of time between applications in a thinned stream of events is determined by the formula of total probability, taking into account (9), (10):

$$
K_{k}(t)=P(A)=\sum_{g=1}^{m} P\left(H_{g}\right) P\left(A / H_{g}\right)=\sum_{g=1}^{m} \pi_{k}^{g} \cdot F_{k, 1, \ldots, g}^{(*) g}(t) .
$$

In the model it is necessary to take into account that some part of the product after the $m$ - measurements remains defective and leaves the system $[15,16]$. The probability that the product after $m$ - measurement will be usable on the basis of formulas (9) is equal to:

$$
p_{k}^{\text {год }}=p \sum_{g=1}^{m}(1-p)^{g-1}=1-(1-p)^{m} .
$$

Accordingly, the probability of rejection is determined from the expression:

$$
p_{k}^{\sigma p}=1-p_{k}^{2 o d}=(1-p)^{m} .
$$

Further, it is necessary to reduce the flow of products coming out from the k-th stage, which will allow us to finally obtain the $\operatorname{FR} K_{k}^{\Sigma}(t)$ of the time between the events in the flow of suitable products coming out from the k-th stage. For this, it is necessary to substitute into the rarefaction formula for the simplest event flow [15] $K_{k}(t), p_{k}^{20 \partial}$ from the expressions (11), (12):

$$
K_{k}^{\Sigma}(t)=p_{k}^{2 o \partial} K_{k}(t)+p_{k}^{2 o \partial} \sum_{i=1}^{\infty}\left(1-p_{k}^{2 o \partial}\right)^{m} K_{k}{ }^{* * i}(t)
$$

Expression (13) allows each of the multiple measurement steps to be replaced by an equivalent stage of single measurements performed by the device, which is the simplest element with two factor states: faulty and efficient and known distribution function of service time determined by (13).

The execution time of all stages will be defined as the sum of the random variables (the times of realization of each stage), and the FR of this time as a composition of the required 
number of distribution laws for the implementation times of each of the steps defined by formula (13).

The mathematical expectations of the times of the k-th stage are equal

$$
m_{k}=\int_{0}^{\infty}\left[1-K_{k}^{\Sigma}(t)\right] d t .
$$

Then the mathematical expectation of the execution time of a task consisting of $\mathrm{n}$ stages $t_{1, n}$ is equal to:

$$
t_{1, n}=\sum_{k=1}^{N} m_{k}
$$

where $m_{k}$ is in (14).

Thus, the constructed semi-Markov model allows to predict the productivity of the technical system on the basis of formulas (13), (14), (16).

Work was supported by the Ministry of Education and Science of the Russian Federation within the framework of the main part of the state order (№ 1.10513.2018/11.12)

\section{References}

1. P.V. Novitsky, Fundamentals of Information Theory of Measuring Devices, (Energia, 1968).

2. P.V. Novitsky, I.A. Zograf, Estimation of errors in measurement results, (Energoatomizdat. Leningr., 1991).

3. RMG 64-2003 GSI. "Providing efficiency in the management of technological processes. Methods and ways to improve the accuracy of measurements»

4. V.Y. Kopp, A.A. Skidan, A.I. Balakin, O.V. Filipovich, Analysis of differential entropy in technical measurements in machine-tool construction, Proceedings of the Odessa Polytechnic University, v. 1 (27), pp. 214-218 (2007).

5. V.Y. Kopp, A.I. Balakin, E.A. Voloshin, A.N. Gadzhibekov, Approaches to controlling the number of multiple two-parameter measurements, Collection of scientific papers SNUNEI, v. 4 (44), pp. 216-221 (2012).

6. V.Y. Kopp, A.A. Skidan, A.I. Balakin, O.V. Filipovich, Optimum estimation of the number of measurements of instrument systems, Collection of scientific papers SNUNEI, v. 1 (21), pp. 231 - 238 (2007).

7. V.Y. Kopp, A.I. Balakin, A.I. Peschansky, E.A. Chuiko, The use of differential entropy for the analysis of multiple high-precision measurements, International Crimean Conference «Microwave \& Telecommunication Technology» (CriMiCo'2013), (2013).

8. V.Y. Kopp, A.I. Balakin, M.V. Zamorenov, Analysis of the applicability of a truncated normal distribution when choosing the optimal number of multiple measurements, v. 6 (2014).

9. V.S. Korolyuk, A.F. Turbine, Stochastic models of systems, (Science. Dumka, 1989).

10. V.S. Korolyuk, A.F. Turbine, Mathematical foundations of phase integration of complex systems, (Science. Dumka, 1978). 
11. V.S. Korolyuk, A.F. Turbin, Markov recovery processes in reliability problems of systems, (Science. Dumka, 1982).

12. V.Y. Kopp, Y.E. Ozherin, A.I. Peschansky, Stochastic models of automated production systems with temporary redundancy, (Published by SevGTU, 2000).

13. V.Y. Kopp, Y.E. Ozherin, A.I. Peschansky, Simulation of automated lines, (Published by SevNTU, 2006).

14. V.Y. Kopp, M.V. Zamorenov, Y.E. Obzherin, O.V. Filipovich, Methods for modeling semi-Markov systems, Adaptive systems of automatic control, v. 1 (26). pp. 208-221 (2015).

15. V.Y. Kopp, A.I. Balakin, A.A. Skidan, K.P. Anikevich, Modeling of control systems with feedbacks, Collection of scientific papers SNUNEI, v. 3 (19), pp. 252-257 (2006).

16. V.Y. Kopp, A.I. Balakin, O.V. Filipovich, Analysis of automated control systems with feedbacks, Optimization of production processes, v. 10, pp. 57-60 (2007). 